\title{
Early Onset Neonatal Sepsis: The Burden of Group B Streptococcal and E. coli Disease Continues
}

AUTHORS: Barbara J. Stoll, MD, a Nellie I. Hansen, MPH, ${ }^{\text {b }}$ Pablo J. Sánchez, MD, ${ }^{\mathrm{c}}$ Roger G. Faix, MD, ${ }^{\mathrm{d}}$ Brenda B.

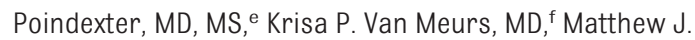

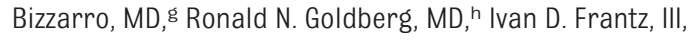
MD, ' Ellen C. Hale, RN, BS, CCRC, , Seetha Shankaran, MD, ${ }^{k}$ Kathleen Kennedy, MD MPH,' Waldemar A. Carlo, MD, m Kristi L. Watterberg, MD, ${ }^{n}$ Edward F. Bell, MD, ${ }^{\circ}$ Michele C. Walsh, MD, MS, ${ }^{p}$ Kurt Schibler, MD, a Abbot R. Laptook, MD, ${ }^{r}$ Andi L. Shane, MD, MPH, MSc, ${ }^{j}$ Stephanie J. Schrag, DPhil, s Abhik Das, PhD, ${ }^{t}$ and Rosemary D. Higgins, MD, u for the Eunice Kennedy Shriver National Institute of Child Health and Human Development Neonatal Research Network

a Emory University School of Medicine, Department of Pediatrics, and Children's Healthcare of Atlanta, Atlanta, Georgia; ${ }^{b}$ Statistics and Epidemiology Unit, RTI International, Research Triangle Park, North Carolina; 'Department of Pediatrics, University of Texas Southwestern Medical Center, Dallas, Texas; dDepartment of Pediatrics, Division of Neonatology, University of Utah School of Medicine, Salt Lake City, Utah; ${ }^{\text {EDepartment of }}$ Pediatrics, Indiana University School of Medicine, Indianapolis,

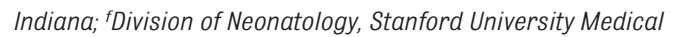
Center, Palo Alto, California; ${ }^{g}$ Department of Pediatrics, Yale University School of Medicine, New Haven, Connecticut; nDepartment of Pediatrics, Duke University, Durham, North Carolina; 'Department of Pediatrics, Tufts Medical Center, Floating Hospital for Children, Tufts Medical Center, Boston, Massachusetts; jDepartment of Pediatrics, Emory University School of Medicine and Children's Healthcare of Atlanta, Atlanta, Georgia; 'Department of Pediatrics, Wayne State University, Detroit, Michigan; 'Department of Pediatrics, University of Texas Medical School at Houston, Texas; 'mDivision of Neonatology, University of Alabama at Birmingham, Birmingham, Alabama; ${ }^{n}$ Department of Pediatrics, University of New Mexico Health Sciences Center, Albuquerque, New Mexico; ${ }^{\circ}$ Department of Pediatrics, University of lowa, lowa City, lowa; ${ }^{p}$ Department of Pediatrics, Rainbow Babies \& Children's Hospital, Case Western Reserve University, Cleveland, Ohio; ' Department of Pediatrics, University of Cincinnati, Ohio; 'rDepartment of Pediatrics, Women \& Infants Hospital, Brown University, Providence, Rhode Island; ${ }^{s}$ Centers for Disease Control and Prevention, Atlanta, Georgia; ${ }^{\text {t}}$ Statistics and Epidemiology Unit, RTI International, Rockville, Maryland; "Eunice Kennedy Shriver National Institute of Child Health and Human Development, Bethesda, Maryland

KEY WORDS

neonatal sepsis, group B streptococcal disease, Escherichia coli infection

(Continued on last page)
WHAT'S KNOWN ON THIS SUBJECT: Early onset neonatal sepsis causes serious morbidity and mortality in newborns. Substantial disease reduction has occurred with intrapartum antibiotic prophylaxis treatment of at-risk women to reduce vertical transmission of early onset group B streptococcal disease. Predominant neonatal pathogens are thought to change over time.

WHAT THIS STUDY ADDS: In a national cohort of $\sim 400000$ infants, rates of early onset sepsis were 0.98 cases per 1000 live births. Infection rates increased with decreasing gestational age and birth weight. The most frequent pathogens were group B streptococci (GBS) in term infants and Escherichia coli in preterm infants. Opportunities for GBS prevention continue to be missed.

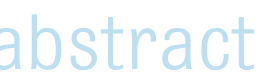

BACKGROUND: Guidelines for prevention of group B streptococcal (GBS) infection have successfully reduced early onset (E0) GBS disease. Study results suggest that Escherichia coli is an important E0 pathogen.

OBJECTIVE: To determine E0 infection rates, pathogens, morbidity, and mortality in a national network of neonatal centers.

METHODS: Infants with E0 infection were identified by prospective surveillance at Eunice Kennedy Shriver National Institute of Child Health and Human Development Neonatal Network centers. Infection was defined by positive culture results for blood and cerebrospinal fluid obtained from infants aged $\leq 72$ hours plus treatment with antibiotic therapy for $\geq 5$ days. Mother and infant characteristics, treatments, and outcomes were studied. Numbers of cases and total live births (LBs) were used to calculate incidence.

RESULTS: Among 396586 LBs (2006-2009), 389 infants developed E0 infection (0.98 cases per $1000 \mathrm{LBs}$ ). Infection rates increased with decreasing birth weight. GBS (43\%, 0.41 per 1000 LBs) and E coli (29\%, 0.28 per $1000 \mathrm{LBs}$ ) were most frequently isolated. Most infants with GBS were term (73\%); $81 \%$ with $E$ coli were preterm. Mothers of $67 \%$ of infected term and $58 \%$ of infected preterm infants were screened for GBS, and results were positive for $25 \%$ of those mothers. Only $76 \%$ of mothers with GBS colonization received intrapartum chemoprophylaxis. Although $77 \%$ of infected infants required intensive care, $20 \%$ of term infants were treated in the normal newborn nursery. Sixteen percent of infected infants died, most commonly with $E$ coli infection (33\%).

CONCLUSION: In the era of intrapartum chemoprophylaxis to reduce GBS, rates of EO infection have declined but reflect a continued burden of disease. GBS remains the most frequent pathogen in term infants, and $E$ coli the most significant pathogen in preterm infants. Missed opportunities for GBS prevention continue. Prevention of $E$ coli sepsis, especially among preterm infants, remains a challenge. Pediatrics 2011;127:817-826 
Early onset sepsis (EOS) in neonates continues to be a serious and feared complication. In the 1970s, group B streptococcal (GBS) infections emerged as the leading cause of EOS and meningitis. ${ }^{1}$ Prevention strategies based on intrapartum chemoprophylaxis to reduce vertical transmission of invasive GBS disease in at-risk women resulted in substantial reduction in early onset GBS disease. ${ }^{2,3}$ In 2002, the Centers for Disease Control and Prevention (CDC) recommended universal antenatal screening at 35 to 37 weeks of pregnancy and intrapartum chemoprophylaxis for women with GBS colonization. ${ }^{4}$ An additional reduction in invasive GBS disease was reported after publication of these revised guidelines. ${ }^{5,6}$

The epidemiology of neonatal sepsis is a changing landscape. ${ }^{7,8}$ In the past decade, several studies revealed increasing rates of early onset Escherichia coli sepsis, particularly among preterm infants. ${ }^{9-14}$ With widespread use of maternal intrapartum antibiotics ( $\sim 30 \%$ of women in the United States receive intrapartum antibiotics for GBS prevention), ${ }^{15}$ concerns have been raised about the potential for increased risk of non-GBS EOS. Because results of several studies demonstrated increased severity of disease and risk of death for newborns with Gram-negative infections, such a change would be particularly alarming. ${ }^{9,16-18}$ Continued surveillance to monitor changes in pathogens, disease severity, and outcome is important as we consider optimal prevention and treatment strategies. In this study we investigated the epidemiology of EO infection among all infants born at centers of the Eunice Kennedy Shriver National Institute of Child Health and Human Development (NICHD) Neonatal Research Network (NRN) during a 4-year period, a cohort of $\sim 400000$ live births (LBs).

\section{METHODS}

Prospective surveillance for EOS and E0 meningitis (EOM) was conducted among live-born infants of all gestational ages (GAs) with birth weights (BWs) $>400 \mathrm{~g}$ who were delivered at 1 of 16 university-based neonatal centers during the period from February 1, 2006, to December 31, 2009. EOS and EOM were defined by isolation of a pathogen from blood or cerebrospinal fluid (CSF) culture of samples drawn within 72 hours of birth and antibiotic treatment for $\geq 5$ days (or death at $<5$ days while receiving antibiotic therapy). Early onset infection included EOS and/or EOM as defined above. Cases were identified by researchteam review of patient, microbiology, and hospital epidemiology records.

Detailed maternal intrapartum and newborn information was collected, including data on maternal GBS screening, intrapartum antibiotic use within 72 hours of delivery, clinical and histologic chorioamnionitis, and maternal risk factors for EO GBS, such as a previous infant with GBS infection, GBS bacteriuria, intrapartum fever $\left(\geq 38.0^{\circ} \mathrm{C}\right)$, delivery at $<37$ weeks, and rupture of membranes $(\mathrm{ROM}) \geq 18$ hours before delivery. Neonatal information included infecting organism, antibiotic therapy, severity of illness, and outcome (death, discharge from hospital). Antibiotic susceptibility data were collected if available.

Coagulase-negative staphylococci (CoNS), micrococci, propionibacteria, corynebacteria, or diphtheroids grown alone in a single culture were considered contaminants, regardless of site determination of contaminant status. Cultures that grew more than 1 organism were considered contaminants on the basis of the attending physician's judgment and decision to discontinue antibiotics before day 5 in a surviving infant. Infants were classified as "symptomatic" if they required blood pressure support at $\leq 72$ hours of age or had respiratory distress, apnea, neutropenia (absolute neutrophil count $<1000 / \mathrm{mm}^{3}$ ) or thrombocytopenia (platelet count $<100000 / \mu \mathrm{L}$ ).

\section{Statistical Analysis}

Maternal and infant characteristics, antibiotic use, and clinical outcomes were examined overall and between infants with $E$ coli and GBS. Statistical significance for unadjusted comparisons was determined by Fisher's exact or $\chi^{2}$ tests (categorical variables) and the Kruskal-Wallis test (continuous variables). Comparisons adjusted for GA were made by using linear or logistic regression models with statistical significance determined by $\mathrm{F}$ or Wald $\chi^{2}$ tests. The adjusted relative risk of death and reported $95 \%$ confidence intervals were based on parameter and variance estimates from a Poisson regression model with robust variance estimators. ${ }^{19}$ Models used data from all infants with EO infection and included GA (continuous) and a pathogen-group indicator, which allowed for comparison of infants with $E$ coli and GBS. Infection rates were determined by the number of infants with EOS/EOM divided by the total number of LBs of infants with a BW of $>400 \mathrm{~g}$ during the study period. BW-specific rates were calculated by using the number of infants with $\mathrm{E} 0$ infection among LBs according to BW group (401-1500 g, 1501-2500 g, and $>2500 \mathrm{~g}$ ).

\section{RESULTS}

Between February 1, 2006, and December 31, 2009, 611 infants with positive cultures of samples obtained at $\leq 72$ hours of age were identified among 396586 infants born at NRN hospitals. A single culture with CoNS was obtained for 149 infants (142 blood cultures and 7 CSF cultures). Some centers considered all CoNS true infections, whereas others considered 
TABLE 1 Pathogens Associated With EOS and EOM

\begin{tabular}{|c|c|c|c|c|}
\hline \multirow[t]{2}{*}{ Pathogen } & \multicolumn{2}{|c|}{$\mathrm{EOS}^{\mathrm{a}}$} & \multicolumn{2}{|c|}{$\mathrm{EOM}^{\mathrm{b}}$} \\
\hline & $n$ & $\%$ & $n$ & $\%$ \\
\hline Gram-positive & 231 & 62 & 8 & 50 \\
\hline GBS & 159 & 43 & 3 & 19 \\
\hline Viridans group streptococci & 20 & 5 & 2 & 13 \\
\hline$S$ aureus ${ }^{c}$ & 9 & 2 & 1 & 6 \\
\hline Enterococci & 10 & 3 & 1 & 6 \\
\hline Group A streptococci & 9 & 2 & - & - \\
\hline Coagulase-negative staphylococcid & 3 & $<1$ & - & - \\
\hline Other Gram-positive ${ }^{\mathrm{e}}$ & 21 & 6 & 1 & 6 \\
\hline Gram-negative & 137 & 37 & 8 & 50 \\
\hline E coli & 107 & 29 & 7 & 44 \\
\hline Haemophili & 11 & 3 & - & - \\
\hline Other Gram-negative ${ }^{f}$ & 19 & 5 & 1 & 6 \\
\hline Fungi & 2 & $<1$ & - & - \\
\hline Candida albicans & 2 & $<1$ & - & - \\
\hline Total & 370 & 100 & 16 & 100 \\
\hline
\end{tabular}

a Data were excluded from 12 infants whose blood culture results were positive for the following polymicrobial infections: GBS + E coli (3); enterococci + CoNS (2); Neisseria species + haemophili (1); Neisseria species + Viridans group streptococci (1); klebsiella + Candida albicans (1); Pseudomonas aeruginosa + Enterobacter species (1); Group A streptococci + CoNS (1); Viridans group streptococci + CoNS (1), Serratia species + enterococci (1)

${ }^{\mathrm{b}}$ Data were excluded from 1 infant with a polymicrobial infection on CSF culture that was considered a true infection by the attending physician (Acinetobacter species + micrococci) and a negative blood culture. Among the remaining 16 infants with EOM, 10 infants had blood and CSF cultures that were positive for the same organism: GBS (2); Viridans group streptococci (1); E coli (7); and 6 infants had negative blood cultures and positive CSF cultures: 1 each for GBS, $S$ aureus, Viridans group streptococci, $\alpha$ streptococci, enterococci, and klebsiella).

${ }^{c}$ One infant with methicillin-resistant $S$ aureus was identified.

${ }^{d}$ These infants had $>1$ positive blood culture for CoNS.

e Organisms found in blood culture: $\alpha$-hemolytic Streptococcus (1); Streptococcus pneumoniae (4); Group D Streptococcus (1); Streptococcus bovis/gallolyticus (4); bacilli (4); Listeria monocytogenes (2); Actinomyces odontolyticus (1); Grampositive rods, CDC group A (1); Gram-positive cocci in chains and pairs (1); non-spore-forming Gram-positive rod (1); aerobic Gram-positive bacilli, not listeria/erysipelothrix/bacillus (1). Organisms found in CSF culture: $\alpha$-hemolytic Streptococcus (1)

f Organisms found in blood culture: Enterobacter species (3); Citrobacter species (2); Acinetobacter species (1); klebsiella (1); Pseudomonas aeruginosa (1); bacteroides (3); Moraxella species (1); Morganella species (5); Capnocytophaga species (2). Organisms found in CSF culture: klebsiella (1).

them all contaminants (range for "true" infections excluding $0 \%$ and 100\%: $14 \%-67 \%)$. Because of striking differences in interpretation, infants for whom a single culture was positive for CoNS were excluded from analysis. Overall, 222 infants had an organism found in culture that was considered a contaminant and were excluded from additional analyses. EOS and/or EOM were diagnosed in 389 infants, of whom 199 (51\%) were preterm (22-36 weeks' GA) and 190 (49\%) were term $(37+$ weeks' GA).

\section{Pathogens and Infection Rates}

The most frequent E0 pathogen was GBS (43\%), followed by E coli (29\%) (Table 1). Infection with Staphylococcus aureus was infrequent; only 1 infant with methicillin-resistant $S$ aureus in blood was identified. No infections with vancomycin-resistant enterococci were identified. Thirteen infants had polymicrobial infections (12 in blood and 1 in CSF). Incidence of EO infection was 0.98 cases per 1000 LBs (range across centers: 0.33-2.44 cases per 1000 LBs). Incidence was highest among infants with a BW of 401 to $1500 \mathrm{~g}$ and lowest among those with a BW of $>2500 \mathrm{~g}$ (Table 2). The overall incidence of infection with GBS (0.41 per 1000 LBs) was higher than E coli (0.28 per 1000 LBs). Rates of $E$ coli infection were higher than rates of GBS among very low birth weight (VLBW) infants (5.09 vs 2.08 per 1000 LBs) and infants with a BW of 1501 to $2500 \mathrm{~g}$ ( 0.54 vs 0.38 per 1000 LBs). Among infants with a BW of $>2500$ g, GBS rates were higher
TABLE 2 Rates of EO Infections per 1000 LBs According to Birth Weight

\begin{tabular}{lcccc}
\hline \multicolumn{5}{c}{ BW, g } \\
\cline { 2 - 5 } & $401-1500^{\mathrm{a}}$ & $1501-2500$ & $>2500$ & All \\
\hline All & 10.96 & 1.38 & 0.57 & 0.98 \\
GBS & 2.08 & 0.38 & 0.35 & 0.41 \\
Ecoli & 5.09 & 0.54 & 0.07 & 0.28 \\
\hline
\end{tabular}

a Rates previously reported by the NRN for VLBW (4011500 g) infants were: 1991-1993: all pathogens 19.3 per 1000 LBs, GBS 5.9 per 1000 LBs, E coli 3.2 per 1000 LBs; 1998-2000: all pathogens 15.4 per 1000 LBs, GBS 1.7 per 1000 LBs, E coli 6.8 per 1000 LBs; 2002-2003: all pathogens 17.0 per 1000 LBs, GBS 1.8 per 1000 LBs, E coli 7.0 per 1000 LBs. ${ }^{9,13}$ Previously reported rates for all pathogens included all treated CoNS; rates for 2006-2009 excluded infants with CoNS except those with $>1$ blood culture positive for CoNS or treated polymicrobial infections involving CoNS. The changes in rates of GBS and $E$ coli between 2002-2003 and 2006-2009 among VLBW infants were not significant.

than $E$ coli rates $(0.35$ vs 0.07 per 1000 LBs).

All infants with $\mathrm{E} 0$ infection had at least 1 blood culture performed (20\% had 1 blood culture; $47 \%$ had 2 blood cultures;, $33 \%$ had $\geq 3$ blood cultures), and $70 \%$ had a lumbar puncture (LP) performed. Likelihood of an LP increased with increasing BW (4011500: 47\%; 1501-2500 g: 71\%; >2500 g: $86 \%)$. Only 17 of 389 infants (4\%; $6 \%$ of those with LP) had a pathogen isolated from CSF; 7 of these infants had a negative blood culture ( $2 \%$ of all infants; 3 of 7 mothers received intrapartum antibiotics). E coli was the most frequently isolated CSF pathogen and was always associated with $E$ coli in blood. Most infants (76\%) were started on antibiotics before their LP, with $\sim 1$ day between blood and CSF cultures.

\section{Antibiotic Susceptibility}

All GBS isolates tested were sensitive to penicillin (93 tested), ampicillin (20 tested), and vancomycin (49 tested); however, $46 \%$ were resistant to erythromycin (63 tested) and $20 \%$ to clindamycin (46 tested). Among $E$ coli isolates tested, $78 \%$ of 102 were ampicillin resistant, 4\% of 103 were gentamicin resistant, and $3 \%$ of 94 
were resistant to third-generation cephalosporins. One E coli isolate resistant to third-generation cephalosporins was reported to be an extended-spectrum $\beta$-lactamase producer. Ampicillin resistance was not more likely among infants whose mothers received intrapartum ampicillin (85\%) than among those who did not $(69 \% ; P=.085)$. Susceptibility results were available for 2 of the 3 infants with CoNS; both were sensitive to vancomycin and gentamicin.

\section{Infant Antibiotic Therapy}

The majority of infants with infections (82\%) received ampicillin and gentamicin only for presumed early infection. EOM was diagnosed in only 17 patients, and they were more likely to be started on ampicillin, gentamicin, and cefotaxime as empiric antibiotic therapy, compared with infants with EOS, ( $24 \%$ vs $4 \% ; P=.008$ ). Small numbers of infants were started on other combinations of antibiotics. After pathogen and susceptibility identification, antibiotics were changed for $44 \%$ infants. Vancomycin, cefotaxime, and penicillin were the antibiotics most frequently added. Among 160 infants with GBS, the majority (73\%) continued on ampicillin once culture results were available; $17 \%$ were switched to penicillin with or without continuation of gentamicin. Among 107 infants with $E$ coli infections, $36 \%$ had initial antibiotic therapy continued while $46 \%$ had cephalosporins added. Among 3 infants with $>1$ blood culture positive for CoNS, 2 had vancomycin added to their empiric therapy. The third infant whose isolate was sensitive to gentamicin remained on gentamicin.

\section{Maternal Intrapartum Antibiotics and GBS Screening}

Overall, $53 \%$ of women who delivered infants with EOS received intrapartum antibiotics (preterm 69\% versus term
$36 \%)$. The proportion of mothers who received antibiotics increased with increasing duration between admission and delivery: $<4$ hours, $37 \% ; 4$ to 24 hours, 46\%; $>24$ hours, 66\%; $P<.001$. Suspected chorioamnionitis (46\%), ROM < 37 weeks' GA (40\%), fever (28\%), and GBS prophylaxis (25\%) were the most common reasons for intrapartum antibiotics, with multiple reasons given more than half the time. When antibiotics were ordered for suspected chorioamnionitis with or without fever, ampicillin (76\%) and gentamicin (71\%) were most frequently given. Ampicillin (77\%) and erythromycin $(46 \%)$ were the most frequently administered antibiotics for ROM $<37$ weeks' GA. GBS prophylaxis alone was the reason for administration of intrapartum antibiotics for 33 of 205 mothers who received antibiotics, with penicillin (52\%) and ampicillin (30\%) most commonly received.

Despite CDC recommendations for universal antenatal screening for GBS, only $67 \%$ of mothers who delivered term infants who had infections were screened; screening was less common among women who delivered preterm infants who had infections (58\%). Among those screened, 25\% were colonized with GBS (Table 3). Of mothers whose infants developed E0 GBS, 58\% were screened (63\% term, $44 \%$ preterm). The GBS screening culture was negative for mothers of $81 \%$ of term and $26 \%$ of preterm infants with E0 GBS, which reflects either a falsenegative screen or a change in colonization status. Only $76 \%$ of mothers with GBS bacteriuria, $76 \%$ with a positive GBS screen, and $66 \%$ with unknown GBS colonization status and a risk factor (gestation $<37$ weeks, ROM $\geq 18$ hours before delivery, maternal temperature $\geq 38.0^{\circ} \mathrm{C}$ ) received intrapartum antibiotics.
Patient Characteristics, Clinical Care, Outcome: GBS Versus $E$ coli Infections

The majority of infants with GBS were term $(73 \%)$, whereas the majority with E coli were preterm (81\%). Infants infected with GBS were more likely than infants with $E$ coli to be black (52\% vs $29 \%$, Table 4). A greater proportion of mothers of infants with EO E coli infection received antibiotics within 72 hours of delivery compared with mothers of infants with GBS ( $79 \%$ vs $38 \%$, adjusted $P<.001$ ).

Chorioamnionitis was more frequently documented in the medical record for mothers of infants with $E$ coli than mothers with GBS infections ( $56 \%$ vs $33 \%$ overall; adjusted $P=.02$, Table 4). Placental pathology analysis was performed for a greater proportion of infants with $E$ colithan GBS ( $81 \%$ vs $52 \%$; adjusted $P=.03$ ). Among 154 women with documented chorioamnionitis, $82 \%$ had placental pathology analysis performed, with histologic chorioamnionitis confirmed for $90 \%$. No difference in the occurrence of histologic chorioamnionitis was detected among infants with GBS or E coli.

Most infants with E0 infection (77\%) required intensive care (95\% preterm, $58 \%$ term infants). However, $20 \%$ of infected term infants seemed healthy (blood culture performed for maternal risk factors) and received care in the normal newborn nursery. Given that more infants with $E$ coli were preterm, it is not surprising that a higher proportion received intensive care $(93 \%)$ compared with infants with GBS (66\%, Table 5). Among infants who received intensive/intermediate care, greater proportions of those with $E$ coli than with GBS had apnea and received blood pressure support, although differences for all except volume expansion were no longer statistically significant after adjustment for GA. Thrombocytopenia was more common 


\begin{tabular}{|c|c|c|c|c|c|}
\hline & \multirow{2}{*}{$\begin{array}{c}\text { All Patients } \\
(N=389)\end{array}$} & \multicolumn{4}{|c|}{ GA of Infants With GBS, wk } \\
\hline & & $\begin{array}{c}22-34 \\
(n=35)\end{array}$ & $\begin{array}{l}35-36 \\
(n=8)\end{array}$ & $\begin{array}{c}37+ \\
(n=117)\end{array}$ & $\begin{array}{c}\text { Overall } \\
(n=160)\end{array}$ \\
\hline \multicolumn{6}{|l|}{ Screened for GBS, $n(\%)$} \\
\hline Yes & $243(62)$ & $15(43)$ & $4(50)$ & $74(63)$ & $93(58)$ \\
\hline No & $96(25)$ & $12(34)$ & $3(38)$ & $34(29)$ & $49(31)$ \\
\hline Unknown & $50(13)$ & $8(23)$ & $1(12)$ & $9(8)$ & $18(11)$ \\
\hline \multicolumn{6}{|l|}{ GBS screen result, $n(\%)^{\text {a }}$} \\
\hline Positive & $60(25)$ & $12(80)$ & $2(50)$ & $14(19)$ & $28(30)$ \\
\hline Negative & $182(75)$ & $3(20)$ & $2(50)$ & $60(81)$ & $65(70)$ \\
\hline Unknown & $1(<1)$ & $0(0)$ & $0(0)$ & $0(0)$ & $0(0)$ \\
\hline \multicolumn{6}{|l|}{ Intrapartum antibiotics, $n(\%)^{\mathrm{b}}$} \\
\hline Received for GBS prophylaxis with or without another indication & $51(13)$ & $5(14)$ & $0(0)$ & $3(3)$ & $8(5)$ \\
\hline Received for other reasons & $154(40)$ & $12(34)$ & $5(63)$ & $36(31)$ & $53(33)$ \\
\hline No intrapartum antibiotics received & $182(47)$ & $18(51)$ & $3(37)$ & $77(66)$ & $98(62)$ \\
\hline \multicolumn{6}{|l|}{ Intrapartum prophylaxis indicated per CDC 2002 guidelines $^{4}$} \\
\hline $\begin{array}{l}\text { Previous infant with GBS, } n \\
\quad \text { Antibiotics received, } n \text { (\%) }\end{array}$ & $\begin{array}{c}1 \\
1(100)\end{array}$ & $0(0)$ & $0(0)$ & $0(0)$ & $0(0)$ \\
\hline Maternal GBS bacteriuria, $n$ & 17 & 3 & 2 & 2 & 7 \\
\hline Antibiotics received, $n$ (\%) & $13(76)$ & $2(67)$ & $2(100)$ & $1(50)$ & $5(71)$ \\
\hline Positive GBS screen (without C-section delivery in the absence of labor or ROM), $n$ & 50 & 10 & 1 & 12 & 23 \\
\hline Antibiotics received, $n(\%)$ & $38(76)$ & $6(60)$ & $0(0)$ & $7(58)$ & $13(57)$ \\
\hline Unknown GBS status, with maternal risk factor, $n^{c}$ & 113 & 20 & 3 & 23 & 46 \\
\hline Antibiotics received, $n(\%)$ & $75(66)$ & $8(40)$ & $2(67)$ & $19(83)$ & $29(63)$ \\
\hline Negative GBS screen $>5$ wk before delivery, no repeat test, delivery at $<37 \mathrm{wk}, n^{\mathrm{d}}$ & 3 & $0(0)$ & 2 & $0(0)$ & 2 \\
\hline Antibiotics received, $n(\%)$ & $2(67)$ & & $1(50)$ & & $1(50)$ \\
\hline \multicolumn{6}{|l|}{ Intrapartum prophylaxis not indicated per CDC 2002 guidelines $^{4}$} \\
\hline Negative GBS screen $\leq 5$ wk before delivery or C-section in the absence of labor or ROM, $n$ & 146 & 2 & $0(0)$ & 47 & 49 \\
\hline Antibiotics received, $n(\%)$ & $65(45)$ & $1(50)$ & & $7(15)$ & $8(16)$ \\
\hline \multicolumn{6}{|l|}{ Other clinical scenarios } \\
\hline $\begin{array}{l}\text { Negative GBS screen }>5 \text { wk before delivery, with maternal risk factor } \\
\quad(\text { excluding delivery at }<37 \mathrm{wk}), n\end{array}$ & 5 & $0(0)$ & $0(0)$ & 2 & 2 \\
\hline Antibiotics received, $n(\%)$ & $2(40)$ & & & $0(0)$ & $0(0)$ \\
\hline Negative GBS screen $>5$ wk before delivery, without maternal risk factor, $n$ & 3 & $0(0)$ & $0(0)$ & 2 & 2 \\
\hline Antibiotics received, $n$ (\%) & $0(0)$ & & & $0(0)$ & $0(0)$ \\
\hline Negative GBS screen, timing unknown, with or without maternal risk factor, $n^{\mathrm{e}}$ & 20 & $0(0)$ & $0(0)$ & 9 & 9 \\
\hline Antibiotics received, $n$ (\%) & $5(25)$ & & & $2(22)$ & $2(22)$ \\
\hline Unknown GBS status, without maternal risk factor or risk factor information missing, $n^{f}$ & 31 & $0(0)$ & $0(0)$ & 20 & 20 \\
\hline Antibiotics received, $n(\%)$ & $4(13)$ & & & $3(15)$ & $3(15)$ \\
\hline
\end{tabular}

a Percents are among mothers screened for GBS.

b Maternal antibiotics received within 72 hours before delivery; information was missing for 2 infants.

${ }^{c}$ Maternal risk factors for EO GBS infection were any of the following: delivery at $<37$ weeks' gestation, R0M $\geq 18$ hours before delivery, and intrapartum fever $\geq 38.0^{\circ} \mathrm{C}$.

${ }^{\mathrm{d}}$ According to the suggested approach for GBS prophylaxis management for women at risk for preterm delivery in the CDC 2002 guidelines, ${ }^{4}$ if delivery has not occurred within 4 weeks of GBS screening, screening should be repeated and the patient managed on the basis of the result (Figure $3^{4}$ ). Because these mothers were not rescreened, GBS status may be considered unknown.

e Among all patients, 9 infants had mothers with 1 or more of the risk factors listed above, 9 were born to mothers with none of the risk factors, and 2 had missing information for maternal risk factors. Among infants who had GBS, 4 had mothers with 1 or more of the specified risk factors, 4 had mothers with none of the risk factors, and 1 had missing information for maternal risk factors.

${ }^{\mathrm{f}}$ Maternal risk factor information was missing for 2 infants among all patients, and for 1 infant among those with GBS.

among infants with $E$ coli than infants with GBS.

Although most infants with EOS/EOM survived to discharge, $16 \%$ died; $57 \%$ deaths were in the first 3 days of life (Table 6). The case fatality rate was inversely related to GA (22-24 weeks: $54 \%$; 25-28 weeks: 30\%; 29-33 weeks: $12 \%$; 34-36 weeks: $0 \%$; $\geq 37$ weeks: $3 \%$ ).
More infants with $E$ coli than with GBS infection died (33\% vs $9 \%$; $P<$ .001). However, after adjustment for GA, the risk of death was not significantly higher for infants with $E$ coli compared with GBS (adjusted relative risk: 1.27 ; $95 \%$ confidence interval: $0.77-2.08)$. Preterm infants with E coli were more likely to have a ful- minant illness with death in the first 3 days of life ( $64 \%$ of those who died).

\section{DISCUSSION}

This prospective surveillance study of almost 400000 live births documents that in the era of intrapartum chemoprophylaxis, the overall rate of E0 infection is low (0.98 per 1000 live 
TABLE 4 Characteristics of Infants with Early-Onset Infections (All Pathogens, GBS, and E colI) and Their Mothers

\begin{tabular}{|c|c|c|c|c|c|c|c|c|}
\hline \multirow[t]{2}{*}{ Infant Characteristics, $n(\%)^{\text {a }}$} & \multirow[t]{2}{*}{$\begin{array}{l}\text { All Patients } \\
(N=389)\end{array}$} & \multicolumn{2}{|c|}{$\begin{array}{l}\text { Preterm (22-36 wk) } \\
\text { With GBS or E coli }\end{array}$} & \multicolumn{2}{|c|}{$\begin{array}{l}\text { Term }(37+\text { wk) With } \\
\text { GBS or E coli }\end{array}$} & \multicolumn{3}{|c|}{ Overall With GBS or E coli } \\
\hline & & $\begin{array}{c}\text { GBS } \\
(n=43)\end{array}$ & $\begin{array}{c}\text { E coli } \\
(n=87)\end{array}$ & $\begin{array}{c}\text { GBS } \\
(n=117)\end{array}$ & $\begin{array}{c}\text { E coli } \\
(n=20)\end{array}$ & $\begin{array}{c}\text { GBS } \\
(n=160)\end{array}$ & $\begin{array}{c}\text { E coli } \\
(n=107)\end{array}$ & $\begin{array}{l}\text { P, Unadjusted } \\
\text { (Adjusted) }^{\mathrm{b}}\end{array}$ \\
\hline Birth weight, g & & & & & & & & $<.001(.4)$ \\
\hline $401-1500$ & 142 (37\%) & $27(63)$ & $66(76)$ & $0(0)$ & $0(0)$ & $27(17)$ & $66(62)$ & \\
\hline $1501-2500$ & $51(13 \%)$ & $11(26)$ & $20(23)$ & $3(3)$ & $0(0)$ & $14(9)$ & $20(19)$ & \\
\hline $2501+$ & $196(50 \%)$ & $5(12)$ & $1(1)$ & $114(97)$ & $20(100)$ & $119(74)$ & $21(20)$ & \\
\hline Gender & & & & & & & & $.2(.1)$ \\
\hline M & $205(53 \%)$ & $22(51)$ & $47(54)$ & $57(49)$ & $15(75)$ & $79(49)$ & $62(58)$ & \\
\hline $\mathrm{F}$ & $184(47 \%)$ & $21(49)$ & $40(46)$ & $60(51)$ & $5(25)$ & $81(51)$ & $45(42)$ & \\
\hline Race/ethnicity & & & & & & & & $.001(.006)$ \\
\hline Black, non-Hispanic & $156(40 \%)$ & $21(49)$ & $24(28)$ & $62(53)$ & $7(35)$ & $83(52)$ & $31(29)$ & \\
\hline White, non-Hispanic & $118(30 \%)$ & $10(23)$ & $34(39)$ & $21(18)$ & $6(30)$ & $31(19)$ & $40(37)$ & \\
\hline Hispanic & $94(24 \%)$ & $10(23)$ & $21(24)$ & $32(27)$ & $6(30)$ & $42(26)$ & $27(25)$ & \\
\hline Other & $21(5 \%)$ & $2(5)$ & $8(9)$ & $2(2)$ & $1(5)$ & $4(3)$ & $9(8)$ & \\
\hline \multicolumn{9}{|l|}{ Maternal characteristics } \\
\hline Mother's age, mean (SD) & $26(6.6)$ & $25(6.0)$ & $28(6.2)$ & $23(5.8)$ & $29(7.2)$ & $24(5.9)$ & $28(6.4)$ & $<.001(<.001)$ \\
\hline Antibiotics within 72 hours before delivery & $205(53)$ & $22(51)$ & $72(84)$ & $39(34)$ & $12(60)$ & $61(38)$ & $84(79)$ & $<.001(<.001)$ \\
\hline $\begin{array}{l}\text { Antenatal steroids } w / \text { in } 72 \text { hours before } \\
\text { delivery }\end{array}$ & $105(27)$ & $17(40)$ & $55(63)$ & NA & NA & NA & NA & $.01(.04)^{\mathrm{c}}$ \\
\hline Type of delivery & & & & & & & & $.02(.1)^{\mathrm{d}}$ \\
\hline Vaginal & $181(47)$ & $18(43)$ & $28(33)$ & $63(54)$ & $9(47)$ & $81(51)$ & $37(35)$ & \\
\hline C-section with labor, with ROM before C/S & $126(33)$ & $15(36)$ & $31(36)$ & $39(33)$ & $7(37)$ & $54(34)$ & $38(36)$ & \\
\hline C-section with labor, without ROM before C/S & $10(3)$ & $0(0)$ & $2(2)$ & $4(3)$ & $1(5)$ & $4(3)$ & $3(3)$ & \\
\hline C-section without labor, with ROM before C/S & $35(9)$ & $4(10)$ & $15(17)$ & $4(3)$ & $1(5)$ & $8(5)$ & $16(15)$ & \\
\hline C-section without labor, without ROM before C/S & $11(3)$ & $2(5)$ & $4(5)$ & $1(<1)$ & $0(0)$ & $3(2)$ & $4(4)$ & \\
\hline C-section, not specified & $22(6)$ & $3(7)$ & $6(7)$ & $6(5)$ & $1(5)$ & $9(6)$ & $7(7)$ & \\
\hline Rupture of membranes $\geq 18$ hours before delivery & $127(33)$ & $19(44)$ & $54(62)$ & $17(15)$ & $4(21)$ & $36(23)$ & $58(55)$ & $<.001(.008)$ \\
\hline $\begin{array}{l}\text { Spontaneous rupture of membranes with or } \\
\text { without labor before } 37 \mathrm{wk}\end{array}$ & $157(40)$ & $30(70)$ & $75(86)$ & NA & NA & NA & NA & $.03(.04)^{\mathrm{e}}$ \\
\hline \multicolumn{9}{|l|}{ Symptoms within 72 hours before delivery } \\
\hline Maternal temperature $\geq 38.0^{\circ} \mathrm{C}$ & $102(26)$ & $3(7)$ & $27(32)$ & $42(36)$ & $6(30)$ & $45(28)$ & $33(31)$ & $.6(.04)$ \\
\hline Uterine or abdominal tenderness & $56(15)$ & $6(14)$ & $26(31)$ & $4(3)$ & $0(0)$ & $10(6)$ & $26(25)$ & $<.001(.06)$ \\
\hline $\begin{array}{l}\text { Foul smelling vaginal discharge or amniotic } \\
\text { fluid }\end{array}$ & $37(10)$ & $3(7)$ & $15(18)$ & $4(3)$ & $0(0)$ & $7(4)$ & $15(14)$ & $.006(.3)$ \\
\hline Maternal tachycardia ( $>100$ beats per min) & $115(30)$ & $14(33)$ & $36(42)$ & $30(26)$ & $7(35)$ & $44(28)$ & $43(41)$ & $.03(.08)$ \\
\hline Fetal tachycardia ( $>160$ beats per min) & $120(31)$ & $11(26)$ & $34(40)$ & $40(34)$ & $7(35)$ & $51(32)$ & $41(39)$ & $.3(.3)$ \\
\hline $\begin{array}{l}\text { Chorioamnionitis documented in the medical } \\
\text { record }\end{array}$ & $154(40)$ & $13(30)$ & $52(61)$ & $39(34)$ & $7(35)$ & $52(33)$ & $59(56)$ & $<.001(.02)$ \\
\hline Placental pathology performed & $248(65)$ & $33(77)$ & $72(85)$ & $49(43)$ & $13(65)$ & $82(52)$ & $85(81)$ & $<.001(.03)$ \\
\hline Histologic chorioamnionitis ${ }^{f}$ & $190(77)$ & $30(91)$ & $63(88)$ & $32(65)$ & $8(62)$ & $62(76)$ & $71(84)$ & $.2(.9)$ \\
\hline
\end{tabular}

Data for 3 infants with both GBS and $E$ coli were included among all patient data but excluded from GBS and $E$ coli columns.

a Among all patients $(N=389)$, information was missing for maternal antibiotics: 2 infants; delivery type: 4; timing of rupture of membranes: 8 ; spontaneous rupture of membranes <37 weeks: 1 ; symptoms within 72 hours before delivery: 4; chorioamnionitis in the medical record: 4; placental pathology: 5 . Among infants with GBS or $E$ coli ( $N=267)$, information was missing for maternal antibiotics: 2 infants; delivery type: 3; symptoms within 72 hours before delivery: 3; chorioamnionitis in the medical record: 3 ; placental pathology: 4.

b $P$ value for a difference between infants overall with GBS versus $E$ coli by Fisher's exact test or the $\chi^{2}$ test for categorical variables, or the Kruskal-Wallis test (mother's age) and adjusting for GA (continuous) in a linear (mother's age) or logistic regression model.

c $P$ value for a difference between infants with GBS versus $E$ coli among preterm infants, unadjusted and adjusting for GA (continuous) in a logistic regression model. No term infants received antenatal steroids.

d $P$ value for a difference between infants with GBS versus $E$ coli in the proportion with c-section versus vaginal delivery.

e $P$ value for a difference between infants with GBS versus $E$ coli among preterm infants, unadjusted and adjusting for GA (continuous) in a logistic regression model.

${ }^{f}$ From among mothers for whom analysis of placental pathology was performed.

births), with the highest rates among the most premature infants. During the 4 year study period, no significant changes were found in the overall rate of EOS or in rates of infection with GBS or E coli, the most frequent pathogens responsible for EOS. GBS remains the most frequent E0 pathogen, especially for term infants. With $\sim 4.3$ million live births in the United States each year, these rates (adjusted for birth weight) reflect an estimated 3306 newborns with EO infection annually and $\sim 344$ neonatal deaths ( $\sim 1619$ cases and 88 deaths with GBS and 751 cases and 194 deaths with $E$ colI), and reveal a continuing burden of disease with morbidity and mortality.

Missed opportunities for prevention of GBS were identified, including failure to screen all women who deliver at 
TABLE 5 Clinical Care and Outcomes for Infants With Early Onset Infections (All Pathogens, GBS, and E coll)

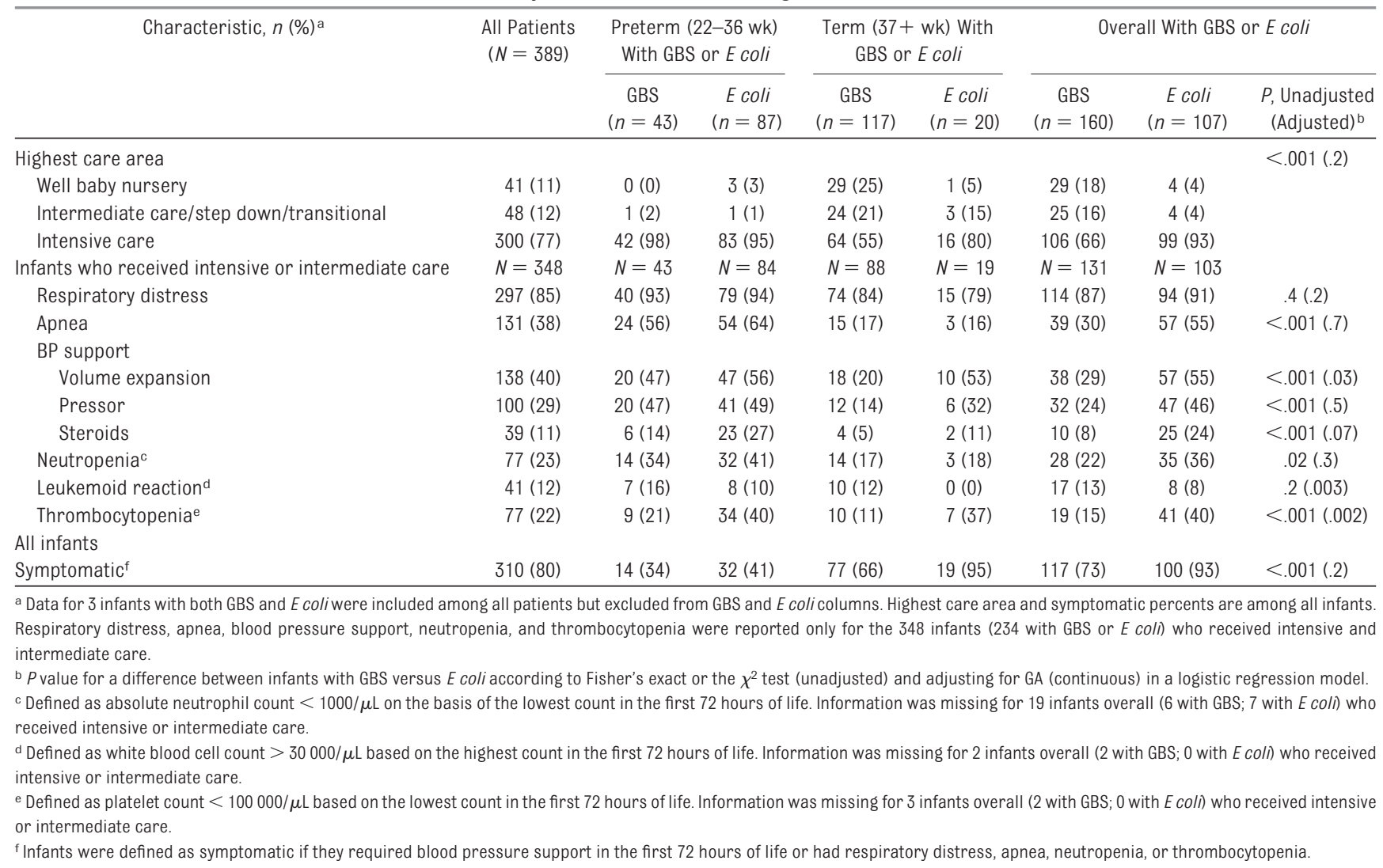

TABLE 6 Mortality and Timing of Death for Infants With Early Onset Infections

\begin{tabular}{|c|c|c|c|c|c|c|c|c|}
\hline & \multirow[t]{2}{*}{$\begin{array}{l}\text { All Patients } \\
(N=389)\end{array}$} & \multicolumn{2}{|c|}{$\begin{array}{l}\text { Preterm (22-36 wk) } \\
\text { With GBS or E coli }\end{array}$} & \multicolumn{2}{|c|}{$\begin{array}{l}\text { Term }(37+\text { wk) With } \\
\text { GBS or E coli }\end{array}$} & \multicolumn{3}{|c|}{ Overall With GBS or E coli } \\
\hline & & $\begin{array}{c}\text { GBS } \\
(n=43)\end{array}$ & $\begin{array}{c}\text { E coli } \\
(n=87)\end{array}$ & $\begin{array}{c}\text { GBS } \\
(n=117)\end{array}$ & $\begin{array}{c}\text { E coli } \\
(n=20)\end{array}$ & $\begin{array}{c}\text { GBS } \\
(n=160)\end{array}$ & $\begin{array}{c}\text { E coli } \\
(n=107)\end{array}$ & $\begin{array}{l}\text { Adjusted RR for } E \text { coli } \\
\text { vs GBS }(95 \% \mathrm{Cl})^{\text {a }}\end{array}$ \\
\hline All deaths, $n(\%)$ & $61(16)$ & $13(30)$ & $33(38)$ & $2(2)$ & $2(10)$ & $15(9)$ & $35(33)$ & $1.27(.77-2.08)$ \\
\hline \multicolumn{9}{|c|}{ Timing of death, $n(\%)^{\mathrm{b}}$} \\
\hline $0-3 d$ & $35(57)$ & $7(54)$ & $21(64)$ & $1(50)$ & $1(50)$ & $8(53)$ & $22(63)$ & \\
\hline $4-7 d$ & $12(20)$ & $1(8)$ & $7(21)$ & $1(50)$ & $1(50)$ & $2(13)$ & $8(23)$ & \\
\hline $8-14 d$ & $3(5)$ & $0(0)$ & $2(6)$ & $0(0)$ & $0(0)$ & $0(0)$ & $2(6)$ & \\
\hline$>14 \mathrm{~d}$ & $11(18)$ & $5(38)$ & $3(9)$ & $0(0)$ & $0(0)$ & $5(33)$ & $3(9)$ & \\
\hline
\end{tabular}

a Relative risk (RR) of death and $95 \%$ confidence interval (CI) for infants with $E$ coli versus GBS infection adjusted for gestational age from a Poisson regression model that included gestational age (continuous) and pathogen group indicator. The adjusted RR was not statistically significant, $P=.35$.

b Percents are among infants who died.

term, failure to provide antibiotics to all colonized women or to those who delivered preterm with unknown colonization status, and false-negative GBS screens among some women who deliver infants with GBS infection. Negative GBS screens among women who deliver infants with GBS are particularly troubling and may be attributable to insufficient sampling, delay in processing, suboptimal laboratory tech- niques, recent antibiotic use, or colonization after screening was performed. Point-of-care diagnostics, ie, accurate rapid GBS diagnostic tests at the time a woman presents in labor, would help reduce the number of colonized women who remain undetected and therefore untreated and would further reduce EO GBS cases. Of note, the recently updated CDC GBS prevention guidelines ${ }^{20}$ recommend chemoprophylaxis for women with risk factors at delivery, despite negative screening cultures.

Several studies have revealed increased rates of $E$ coli infection among preterm infants. ${ }^{8,9}$ The results of our study, conducted in a large and geographically diverse population, establish that $E$ coli has become the most frequent EOS pathogen among preterm infants and that preterm infants 
with $E$ coli infection are at highest risk of severe disease and death. The NICHD NRN has been studying infections among VLBW infants for almost 2 decades. A major shift in EOS pathogen distribution among VLBW infants was identified at NRN sites in the late 1990s (reduction in GBS and increase in $E$ coll) ${ }^{9,13}$ Although the results of the current study confirm these earlier observations, it is reassuring that the incidence of $E$ coli among VLBW infants at NRN sites has not continued to increase and $E$ coli has not emerged as a frequent $E 0$ pathogen among term infants. Our data suggest that widespread use of intrapartum antibiotic prophylaxis to reduce vertical transmission of GBS has not resulted in a further increase in non-GBS E0 sepsis among the larger cohort of infants of all BWs or among VLBW infants beyond that noted previously. Prevention strategies for E0 E coli disease need to be developed and implemented. Analysis of risk factors associated with $\mathrm{EO} E$ coli (as was done with GBS) could help identify high risk women to target for prevention. Our data suggest that severe manifestations of clinical chorioamnionitis (uterine tenderness, foulsmelling amniotic fluid) may be markers for increased risk of $E$ coli infection and warrant broad spectrum antibiotic therapy in the mother and infant.

Strengths of this study include the size of the NRN and the detailed information collected prospectively, including clinical data collection beyond the scope of most surveillance systems. Although the NRN is a large and diverse national network of academic centers and we reviewed infection status of almost 400000 LBs, this is not a population-based nationally representative cohort. Other limitations include review of case data only, without an uninfected comparison group to assess risk factors and GBS prevention strategies more broadly. E0 infection was defined by positive cultures. Because of widespread use of intrapartum antibiotics, some infants with true, but culture-negative infection would not have been identified in this study, a limitation of all studies that base EOS rates on newborn blood culture results.

Bacteremia is a known risk factor for neonatal meningitis. ${ }^{21-23}$ In our cohort, only two-thirds of infants with EOS underwent an LP. More term infants underwent an LP, in large part because preterm infants are often considered too unstable to tolerate an LP. Although most CSF cultures were negative, $76 \%$ of infants had their LP performed after antibiotics had been initiated, which may have sterilized their CSF and made meningitis more difficult to diagnose. Although the number of infants with a positive CSF culture and a negative blood culture was low, these cases are a reminder that meningitis will be missed in some infants if an LP is not part of the sepsis workup. ${ }^{23}$ Moreover, antibiotic therapy may change with EOM caused by Gramnegative organisms.

The importance of CoNS as EOS pathogens remains unclear. Although 149 infants had a single early culture that was positive for CoNS, more than half of these were judged to be contaminants by the reporting study center, with wide center variability in assessment of a single positive blood culture. Only 3 infants had $>1$ positive CoNS culture and what was considered to be a true infection. Prospective studies to investigate the vaginal ecology of pregnant women and the vertical transmission of CoNS, as well as continued surveillance for these organisms among newborns with EOS, will help determine if CoNS are emerging as important EO pathogens.

Rational choice of antibiotics for the infant with presumed infection re- quires review of antibiotic susceptibility of the predominant organisms that cause disease at the local level. ${ }^{24}$ Treatment of the infected infant must be based on antibiotic susceptibility of the infecting organism and clinical outcome. Ampicillin and gentamicin continue to be the recommended empiric antibiotics for the infant at risk for EOS. ${ }^{25}$ Our study results support this recommendation, because all GBS isolates tested were sensitive to ampicillin and $96 \%$ of $E$ coli isolates tested were sensitive to gentamicin.

With neonatal intensive care, the vast majority of infants with EOS survive. The most immature infants remain at highest risk of death. Although some investigators have reported higher mortality for infants infected with Gram-negative agents, after we adjusted for GA we did not find a statistically significant increase in the risk of death for infants infected with $E$ coli versus GBS. Although improved treatment strategies may reduce the current $16 \%$ mortality, strategies to reduce the unacceptably high rates of preterm birth in the United States are more likely to reduce deaths associated with early onset infections, as well as deaths related to other complications of prematurity.

\section{ACKNOWLEDGMENTS}

This study was supported in part by PHS grant UL1 RR025008 from the Clinical and Translational Science Award program, National Institutes of Health, National Center for Research Resources. The National Institutes of Health, the NICHD, and the Centers for Disease Control and Prevention provided grant support for the Neonatal Research Network's Early Onset Sepsis Study. Data collected at participating sites of the NICHD NRN were transmitted to RTI International, the data coordinating center (DCC) for the network, which stored, managed, and analyzed the data for this study. On behalf of the 
NRN, Dr Abhik Das (DCC Principal Investigator), and Ms Nellie Hansen (DCC Statistician) had full access to all the data in the study and take responsibility for the integrity of the data and accuracy of the data analysis.

The following investigators, in addition to those listed as authors, participated in this study: NRN Steering Committee Chair: Michael S. Caplan, MD, University of Chicago, Pritzker School of Medicine; Case Western Reserve University Rainbow Babies \& Children's Hospital (GCRC M01 RR80, U10 HD21364): Avroy A. Fanaroff, MD, Nancy S. Newman, BA, RN; CDC (IAA 05FED32885-00): Stephanie J. Schrag, DPhil; Cincinnati Children's Hospital Medical Center, University of Cincinnati Hospital, and Good Samaritan Hospital (GCRC M01 RR8084, U10 HD27853): Edward F. Donovan, MD, Kate Bridges, MD, Barbara Alexander, RN, Cathy Grisby, BSN, CCRC, Holly L. Mincey, RN, BSN, Jody Hessling, RN; Duke University School of Medicine University Hospital, Alamance Regional Medical Center, and Durham Regional Hospital (GCRC M01 RR30, U10 HD40492): C. Michael Cotten, MD, MHS, Kathy J. Auten, MSHS, Kimberly A. Fisher, PhD, FNP-BC, IBCLC, Katherine A. Foy, RN; Emory University Children's Health Care of Atlanta, Grady Memorial Hospital, and Emory Crawford Long Hospital (U10 HD27851, 5P20RR11104): David P. Carlton, MD, Ann M. Blackwelder, RNC, BS, MS; NICHD: Stephanie Wilson Archer, MA; Indiana University, Indiana University Hospital, Methodist

\section{REFERENCES}

1. McCracken GH Jr. Group B streptococci: The new challenge in neonatal infections. $J P e$ diatr. 1973;82(4):703-706

2. Schrag SJ, Zywicki S, Farley MM, et al. Group B streptococcal disease in the era of intrapartum antibiotic prophylaxis. NEng/ J Med. 2000;342(1):15-20

3. Centers for Disease Control and Prevention (CDC). Early onset and late-onset neonatal group B streptococcal disease:
Hospital, Riley Hospital for Children, and Wishard Health Services (GCRC M01 RR750, U10 HD27856): Dianne E. Herron, RN, Leslie Dawn Wilson, BSN, CCRC; RTI International (U01 HD36790): W. Kenneth Poole, PhD, Jeanette O'Donnell Auman, BS, Margaret Cunningham, BS, Carolyn Petrie Huitema, MS, Kristin M. Zaterka-Baxter, RN, BSN; Stanford University Dominican Hospital, El Camino Hospital, and Lucile Packard Children's Hospital (GCRC M01 RR70, U10 HD27880): David K. Stevenson, MD, Marian M. Adams, MD, Magdy Ismail, MD, MPH, M. Bethany Ball, BS, CCRC, Andrew W. Palmquist, RN, Melinda S. Proud, RCP; Tufts Medical Center Floating Hospital for Children (GCRC M01 RR54, U10 HD53119): Brenda L. MacKinnon, RNC, Ellen Nylen, RN, BSN; University of Alabama at Birmingham Health System and Children's Hospital of Alabama (GCRC M01 RR32, U10 HD34216): Namasivayam Ambalavanan, MD, Monica V. Collins, RN, BSN, MAEd, Shirley S. Cosby, RN, BSN; University of lowa Children's Hospital (GCRC M01 RR59, U10 HD53109): John A. Widness, MD, Karen J. Johnson, RN, BSN; University of New Mexico Health Sciences Center (GCRC M01 RR997, U10 HD53089): Conra Backstrom Lacy, RN, Rebecca Montman, BSN; University of Texas Southwestern Medical Center at Dallas Parkland Health \& Hospital System and Children's Medical Center Dallas (GCRC M01 RR633, U10 HD40689): Charles R. Rosenfeld, MD, Walid A. Salhab, MD, Gaynelle Hensley, RN, Melissa H. Leps,

United States, 1996-2004. MMWR Morb Mortal Wkly Rep. 2005;54(47): 1205-1208

4. Schrag S, Gorwitz R, Fultz-Butts K, Schuchat A. Prevention of perinatal group B streptococcal disease. Revised guidelines from CDC. MMWR Recomm Rep. 2002;51(RR-11): $1-22$

5. Phares CR, Lynfield R, Farley MM, et al. Epidemiology of invasive group $B$ streptococ-
RN, Nancy A. Miller, RN, Alicia Guzman; University of Texas Health Science Center at Houston Medical School, Children's Memorial Hermann Hospital, and Lyndon Baines Johnson General Hospital/Harris County Hospital District (U10 HD21373): Kathleen A. Kennedy, MD, MPH, Georgia E. McDavid, RN, Patti L. Tate, RCP, Sharon L. Wright, MT; University of Utah University Hospital, LDS Hospital, and Primary Children's Medical Center (CTSA UL1 RR25764, GCRC M01 RR64, U10 HD53124): Bradley A. Yoder, MD, Karen A. Osborne, RN, BSN, CCRC, Jennifer J. Jensen, RN, BSN, Cynthia Spencer, RNC, Kimberlee Weaver-Lewis, RN, BSN; Wayne State University Hutzel Women's Hospital and Children's Hospital of Michigan (U10 HD21385), Rebecca Bara, RN, BSN; Women \& Infants' Hospital of Rhode Island, Warren Alpert Medical School at Brown University (U10 HD27904): William Oh, MD, Angelita M. Hensman, RN, BSN; Yale University Yale-New Haven Children's Hospital and Bridgeport Hospital (CTSA UL1 RR24139, GCRC M01 RR125, GCRC M01 RR6022, U10 HD27871): Richard A. Ehrenkranz, MD, Harris Jacobs, MD, Patricia Cervone, RN, Monica Konstantino, RN, BSN, JoAnn Poulsen, RN, Janet Taft, RN, BSN.

We are indebted to our medical and nursing colleagues and the infants and their parents who agreed to take part in this study. We thank Dr Jennifer Verani for technical advice regarding GBS guidelines and Ms Mazie Tinsley for manuscript preparation.

cal disease in the United States, 1999-2005. JAMA. 2008;299(17):2056-2065

6. Van Dyke MK, Phares CR, Lynfield R, et al. Evaluation of universal antenatal screening for group B streptococcus. N Engl J Med. 2009;360 (25):2626-2636

7. Bizzarro MJ, Raskind C, Baltimore RS, Gallagher PG. Seventy-five years of neonatal sepsis at Yale: 1928-2003. Pediatrics. 2005; 116(3):595-602 
8. Bizzarro MJ, Dembry L-M, Baltimore RS, Gallagher PG. Changing patterns in neonatal Escherichia colisepsis and ampicillin resistance in the era of intrapartum antibiotic prophylaxis. Pediatrics. 2008;121(4): 689-696

9. Stoll BJ, Hansen N, Fanaroff AA, et al. Changes in pathogens causing early-onset sepsis in very-low-birth-weight infants. N Engl J Med. 2002;347(4):240-247

10. Cordero L, Rau R, Taylor D, Ayers LW. Enteric Gram-negative bacilli bloodstream infections: 17 years' experience in a neonatal intensive care unit. Am J Infect Control. 2004;32(4):189-195

11. Daley AJ, Isaacs D, and the Australasian Study Group for Neonatal Infections. Tenyear study on the effect of intrapartum antibiotic prophylaxis on early onset group B streptococcal and Escherichia colineonatal sepsis in Australasia. Pediatr Infect Dis J. 2004;23(7):630-634

12. Jones B, Peake K, Morris AJ, McCowan LM, Battin MR. Escherichia coli: a growing problem in early onset neonatal sepsis. Aust N Z J Obstet Gynaecol. 2004;44(6):558-561

13. Stoll BJ, Hansen NI, Higgins RD, et al. Very low birth weight preterm infants with early onset neonatal sepsis: the predominance of Gram-negative infections continues in the National Institute of Child Health and Human Development Neonatal Research Network,
2002-2003. Pediatr Infect Dis J. 2005;24(7): 635-639

14. Schrag SJ, Hadler JL, Arnold KE, MartellCleary P, Reingold A, Schuchat A. Risk factors for invasive, early-onset Escherichia coli infections in the era of widespread intrapartum antibiotic use. Pediatrics. 2006; 118(2):570-576

15. Schrag SJ, Zell ER, Lynfield R, et al. A population-based comparison of strategies to prevent early-onset group B streptococcal disease in neonates. N Engl J Med. 2002; $347(4): 233-239$

16. Karlowicz MG, Buescher ES, Surka AE. Fulminant late-onset sepsis in a neonatal intensive care unit, 1988-1997, and the impact of avoiding empiric vancomycin therapy. Pediatrics. 2000;106(6):1387-1390

17. Mayor-Lynn K, González-Quintero VH, O'Sullivan MJ, Hartstein Al, Roger S, Tamayo M. Comparison of early onset neonatal sepsis caused by Escherichia coli and group B Streptococcus. Am J Obstet Gynecol. 2005; 192(5):1437-1439

18. Cohen-Wolkowiez M, Moran C, Benjamin DK, et al. Early and late onset sepsis in late preterm infants. Pediatr Infect Dis J. 2009; 28(12):1052-1056

19. Zou G. A modified Poisson regression approach to prospective studies with binary data. Am J Epidemiol. 2004;159(7):702-706

20. Verani JR, McGee L, Schrag SJ; Division of
Bacterial Diseases, National Center for Immunization and Respiratory Diseases, Centers for Disease Control and Prevention (CDC). Prevention of perinatal group B streptococcal disease: revised guidelines from CDC, 2010. MMWR Recomm Rep. 2010; 59(RR-10):1-32

21. Stoll BJ, Hansen N, Fanaroff, et al. To tap or not to tap: high likelihood of meningitis without sepsis among very low birth weight infants. Pediatrics. 2004;113(5):1181-1186

22. Ansong A, Smith PB, Benjamin D, et al. Group B streptococcal meningitis: cerebrospinal fluid parameters in the era of intrapartum antibiotic prophylaxis. Early Hum Dev. 2009; 85(10 suppl):S5-S7

23. Garges HP, Moody A, Cotten CM, et al. Neonatal meningitis: what is the correlation among cerebrospinal fluid cultures, blood cultures, and cerebrospinal fluid parameters? Pediatrics. 2006;117(4):1094-1100

24. Bromiker R, Arad I, Peleg 0, Preminger A, Engelhard D. Neonatal bacteremia: patterns of antibiotic resistance. Infect Control Hosp Epidemiol. 2001;22(12):767-770

25. American Academy of Pediatrics. In: Pickering LK, Baker CJ, Kimberlin DW, Long SS, eds. Red Book: 2009 Report of the Committee on Infectious Diseases. 28th ed. Elk Grove Village, IL: American Academy of Pediatrics; 2009

(Continued from first page)

www.pediatrics.org/cgi/doi/10.1542/peds.2010-2217

doi:10.1542/peds.2010-2217

Accepted for publication Jan 11, 2011

Address correspondence to Barbara J. Stoll, MD, Department of Pediatrics, Emory University School of Medicine, and Children's Healthcare of Atlanta, 2015 Uppergate Drive, Atlanta, GA. E-mail: barbara_stoll@oz.ped.emory.edu

PEDIATRICS (ISSN Numbers: Print, 0031-4005; Online, 1098-4275).

Copyright (C) 2011 by the American Academy of Pediatrics

FINANCIAL DISCLOSURE: The authors have indicated they have no financial relationships relevant to this article to disclose.

Funded by the National Institutes of Health (NIH). 